\title{
Two-dimensional Polymers in Microscopy and Spatially Resolved Vibrational Analysis - A Review
}

\author{
Timo S. G. Niepel, Yashashwa Pandey, and Renato Zenobi*
}

\begin{abstract}
Two-dimensional polymers comprise a novel class of macromolecules with promising mechanical and chemical properties that have recently become accessible under mild synthetic conditions. As nanometer-sized holes and defects are likely to influence these materials' properties, there is a need for analytical methods to spatially and chemically characterize them down to the nanoscale. In this article, we compare tip-enhanced Raman spectroscopy to common methods in nanoanalysis for the investigation of defect sites in molecularly thin 2D-polymer sheets and briefly present measures to meet the challenges arising from tip-induced degradation processes.
\end{abstract}

Keywords: 2D polymer $\cdot$ AFM $\cdot$ Nanomaterials $\cdot$ Spectroscopy $\cdot$ STM $\cdot$ TERS

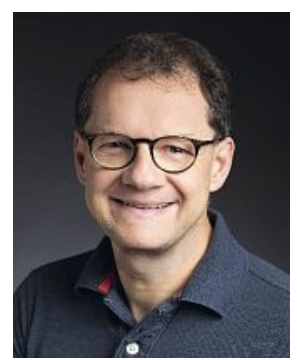

Renato Zenobi is Professor of Analytical Chemistry at the Swiss Federal Institute of Technology (ETH) Zurich. After a PhD at Stanford University and postdoctoral appointments at the University of Pittsburgh and at the University of Michigan, he started his independent career in 1992 as a Werner Fellow at EPF Lausanne. He joined ETH as an assistant professor in 1995 and was promoted to full professor in 2000. Zenobi has made important contributions to the understanding of the ion formation mechanism in matrix-assisted laser desorption/ionization (MALDI) mass spectrometry, to the development of analytical tools for the nanoscale, in particular tip-enhanced Raman spectroscopy (TERS), and to exhaled breath analysis using ambient ionization methods.

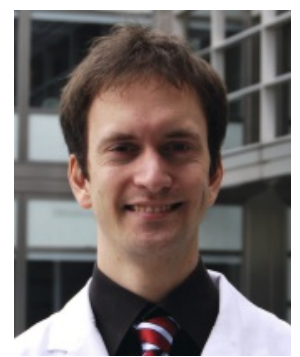

Timo Sami Georg Niepel studied chemistry at the RWTH Aachen University, Germany, joining Carsten Bolm's group for his 2012 bachelor's thesis on iron(VI) compounds when he was admitted into the German National Academic Foundation. After a research stay at the Max Planck Institute for Chemistry, Mainz, he wrote his Master's thesis with Gerhard Raabe and Ken Houk at the University of California, Los Angeles, computationally elucidating the mechanism of Robert Grubbs' ring-opening metathesis polymerization. He then started working at the ETH Zurich in 2015, where he is now a doctoral student in Renato Zenobi's lab.

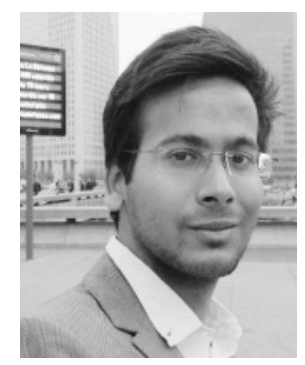

Yashashwa Pandey obtained an Integrated MTech degree in Industrial Chemistry from IIT (BHU), Varanasi, India in 2017. He pursued his Master's project at IBM Research - Zurich, working with Emanuel Lörtscher in collaboration with Hatice Altug at EPF Lausanne, and continued this project as a postgraduate researcher. He worked on the fabrication of dielectric metasurfaces for biosensing applications and grating ordercoupled nanogap antennas for surface-enhanced infrared spectroscopy using electron beam lithography. He later joined the group of Renato Zenobi at ETH Zurich for his PhD in TERS on sensitive 2D-materials.

\section{Introduction}

Over the past decades, the rapid advances in synthetic polymer chemistry have made available many techniques and reaction designs for macromolecules of various physicochemical properties, architectures and dispersities. ${ }^{[1,2]}$ Many modern approaches to engineer a macromolecular material showing the desired thermal and mechanical behavior make use of branching sites at which orthogonal functionalities of the monomeric units act as anchors for the growth ${ }^{[3]}$ or the attachment ${ }^{[4]}$ of side chains. ${ }^{[5]}$ It is these branching sites, induced by the presence of multiple reactive centers on a monomer molecule, ${ }^{[1 b]}$ that enable the formation of macromolecular covalent networks in two and three dimensions; the resulting three-dimensional materials enjoy widespread applications in everyday life. ${ }^{[6]}$ Just like materials extending in three dimensions which consist of one-dimensional polymer molecules would be seen as 1D-materials, two-dimensional polymers may also stack vertically yielding a bulk crystal of 2D-material extending in three dimensions.

The key feature that distinguishes a two-dimensional polymer from other two-dimensional covalent networks and covalent (metal-)organic frameworks is the two-dimensional constitutional repeat unit that seamlessly tiles the plane. A 2D-polymer is thus by definition a monolayer and crystalline, though a small number of defects may be tolerated as long as they do not dominate the 
macromolecule's overall physicochemical properties. On the contrary, 2D-materials may be devoid of repeat units, perhaps even be composed of multiple layers of considerable overall thickness, as long as their sheet-like constituents are not chemically interconnected.

Prominent examples for these multi-layered 2D-materials include graphite, phyllosilicates, chromium(III) halides and transition metal dichalcogenides such as $\mathrm{MoS}_{2}$. Individual two-dimensional monolayers (e.g. graphene) have been obtained from them inter alia via exfoliation and solvothermal processes. ${ }^{[7]}$

Rather new is the bottom-up synthesis based on an in-plane polymerization of an isolated monolayer of multifunctional organic monomers. These novel two-dimensional polymer sheets are conveniently prepared on the air-water interface of a LangmuirBlodgett trough, ${ }^{[8]}$ where the monomers are first compressed to a monolayer and then polymerized by the addition of a reactant or by irradiating the surface. ${ }^{[9]}$ Examples for such structures are shown in Figs. 1a-d. The corresponding multi-layered 2D-materials can also be synthesized in bulk from their monomers and they have been well characterized, ${ }^{[10]}$ however, only small coherent 2D-polymer sheets could be obtained from their bulk crystals by exfoliation. Despite the attractive ease of creating crystals of stacked layers in this way, what drives research on regular covalent monolayers is their promising mechanical properties which render them intriguing new candidates for homogeneous nanoporous membrane materials. In that sense, the interfacial synthesis is vastly superior for the generation of $2 \mathrm{D}$-polymers of sizes needed for their potential industrial applications. Process control now calls for procedures to characterize these ultra-thin films both spatially and chemically. This review will hence focus on analytical methods suitable for the study of fully synthetic organic two-dimensional polymer monolayers, with an emphasis on those that have been successfully applied on samples of 2D-polymers synthesized using surface film techniques.

\section{Methods and Results}

Traditionally, a number of orthogonal analytical techniques are applied to a given sample in order to characterize it chemically and either determine or confirm its identity. This is also the case for thin films, where certain methods such as optical microscopy, electron microscopy, diffraction, scanning probe microscopy (SPM) and tip-enhanced Raman spectroscopy (TERS) have become established standards. Though theoretically possible and potentially useful, not all varieties of these methods have, as of yet, been applied to synthetic 2D-polymer samples. The following sections will briefly discuss the aforementioned techniques' specialties, strengths and weaknesses and assess what information they can provide on samples of 2D-polymers.

\subsection{Optical Microscopy}

Known since the $18^{\text {th }}$ century and probably one of the most common and easy-to-use techniques, optical microscopy allows one to view samples enlarged by the magnification of a lens, with typical length scales ranging from fractions of a millimeter to larger areas, centimeters across. As monolayers are close to invisible in an upright incident light microscope due to their extremely small thickness, this method can usually only serve as a swift control for the exclusion of large or colored contaminants or visible mechanical damage to the sample (e.g. scratches). Nonetheless, efforts have been made to adapt optical microscopy to the investigation of thin layered sheets, improving the contrast by making use of the sample's fluorescent properties or interference effects (cf. Fig. 2a). ${ }^{[15]}$ Related techniques such as frequency dependent transmittance and reflectance, ${ }^{[16]}$ picometrology, ${ }^{[17]}$ spectroscopic ellipsometry imaging, ${ }^{[18]}$ spatial light interference microscopy (SLIM), ${ }^{[19]}$ quadriwave lateral shearing interferometry (QLSI), ${ }^{[20]}$ optical path density measurements (OPD) and Brewster angle mi-
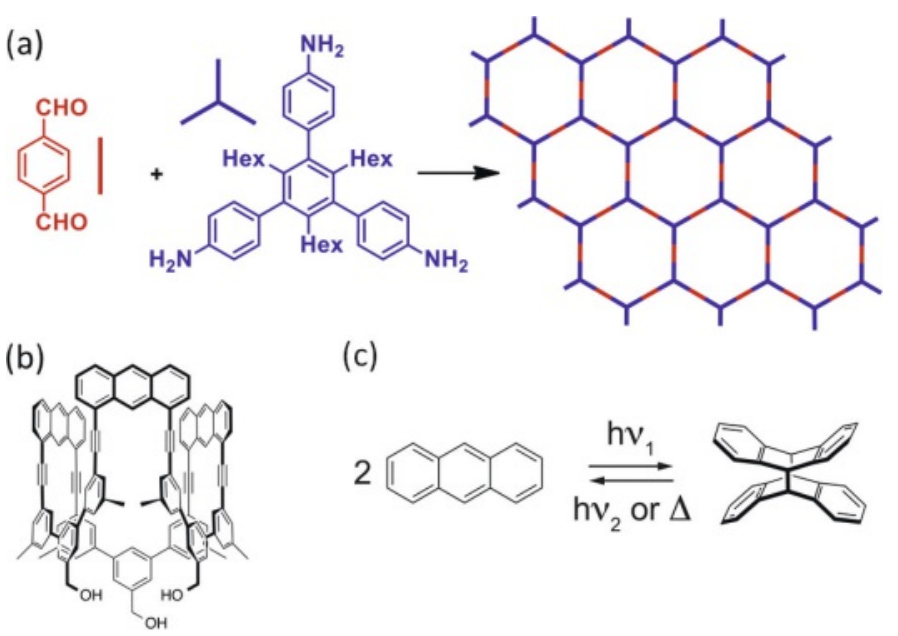

(c)

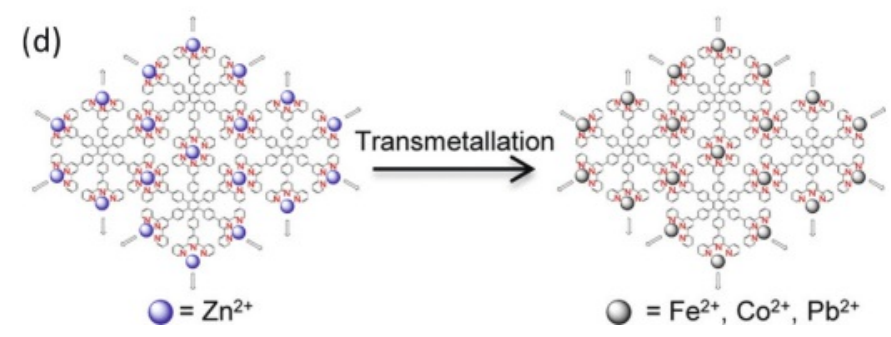

Fig. 1: Prominent synthetic pathways for two-dimensional polymers. ${ }^{[11]}$ a) Dialdehydes condense with triamines forming an imine network in 2D. ${ }^{[12]} \mathrm{b}-\mathrm{c}$ ) The monomer's three anthracene moieties undergo a photochemical [4 + 4]-cycloaddition. These amphiphilic monomers can be dispersed at an air-water interface, with the alcoholic functions preferably pointing downwards, into the aqueous phase. ${ }^{[13]}$ d) Hexafunctional terpyridine ligands chelate and exchange heavy metal cations at the liquid boundary. ${ }^{[14]}$

croscopy (BAM) have been applied to thin films. Among these, OPD offers a high sensitivity and is capable of visualizing samples with thicknesses down to the sub-nanometer range. ${ }^{[20]}$ This performance makes it comparable to the profile information available from scanning probe methods, however with a diffraction limited lateral resolution. For the study of 2D-polymers, BAM has found an application in determining the size and relative arrangement of sheet fragments. ${ }^{6]}$ BAM measurements can be carried out in real time, which allows for observing structural changes in thin films during their preparation. Schlüter and coworkers ${ }^{[6]}$ have used BAM to record the contraction and 'tearing' of a Langmuir film of monomer molecules upon irradiation with light, which they interpreted as a sign of polymerization.

\subsection{Electron Microscopy and Diffraction}

Because of its versatility and high lateral resolving power even below $50 \mathrm{pm}$, electron microscopy ${ }^{[23]}$ has become a popular tool for the investigation of miscellaneous surfaces. In the study of thin samples, high-resolution transmission electron microscopy (HRTEM) ${ }^{[24]}$ is often combined with the acquisition of the electrons' diffraction pattern (selected area electron diffraction, SAED), allowing to reveal regular lateral patterns in the sample. This is of high interest for crystalline 2D-materials since the regularity of their repeat units would be expected to yield distinct reflections from which structural information can be extracted. However, TEM/SAED of 2D-polymers presents a significant challenge. Ultra-thin samples of low thermal and electrical conductivity suffer from rapid degradation, if not outright destruction, when exposed to the electron beam, even under cryogenic conditions ( $c f$. Fig. 2b). ${ }^{[15]}$ In an effort to reduce radiative damage to the sample and enhance its resilience, Algara-Siller et al. ${ }^{[25]}$ have encapsulated a two-dimensional polymer between two sheets of 

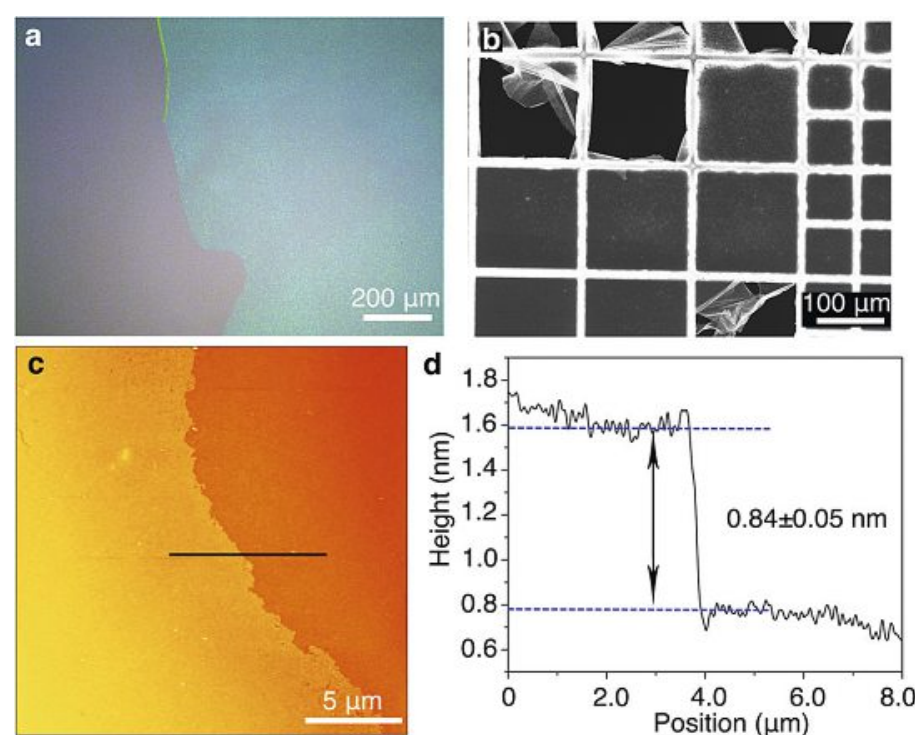

Fig. 2: A 2D-polymer sheet (cf. Fig. 1a) was examined using various microscopic techniques. ${ }^{[21,22]}$ a) By optical microscopy, the polymer monolayer is distinguishable from the bare substrate by a slight difference in color. b) The SEM image shows that the 2D-polymer sheet is sturdy enough to span the gaps in a TEM grid, but suffers damage from the incident electron beam. $\mathrm{c}, \mathrm{d}) \mathrm{A}$ much higher lateral resolution is achieved in AFM, which reveals that the covalent sheet is a monolayer.

graphene and restricted themselves to low electron doses. In this fashion, they were able to preserve the polymer's internal structure and record TEM images and a SAED pattern showing several rings composed of weak but clear lattice reflections.

Another interesting approach to the structural elucidation of $2 \mathrm{D}$-materials has recently been presented by Hofer et al. ${ }^{[10]}$. They directly polymerized single crystals of a suitable monomer by irradiating them with light and confirmed the conversion to the corresponding layered polymer sheets by means of X-ray diffraction followed by a structure refinement. Although no 2D-polymers were isolated in this study, the authors were able to conclude that mechanical strain due to a smaller lattice constant in the polymer sheets does influence the kinetics of polymerization, in good agreement with the familiar 'tearing' observed in LangmuirBlodgett films of monomers upon their photopolymerization. ${ }^{[6]}$

\subsection{Scanning Probe Microscopy}

The backscattering of photons, electrons and other particles is not the only way of creating an image of a sample. The topography of conductive surfaces, for example, can be studied with excellent accuracy by means of scanning tunneling microscopy (STM). [26] This technique, which was invented by Binnig and Rohrer at the beginning of the 1980s at the IBM Zurich Research Lab in Rüschlikon, Switzerland, relies on a minute current of electrons tunneling through a gap between the sample surface and a sharp tip that is very strongly distance dependent. Sweeping this tip over the surface line by line yields a topographical image with down to nanometer resolution - shortly after, Binnig and Rohrer were awarded the Nobel Prize in Physics for their invention, together with Ruska for his development of the electron microscope.

Since the tunneling process of electrons is not particularly hindered by a nanometer-thin spacer of non-conductive material, two-dimensional polymers on conductive substrates such as gold have successfully been imaged topographically by using STM imaging. ${ }^{[9]}$

Quite similarly, atomic force microscopy (AFM) ${ }^{[27]}$ relies on the interaction of a scanning tip with the sample surface. Instead of measuring a tunneling current, it detects the weak attractive and repulsive forces created by the interaction of the molecular orbitals of the analyte with an approached probe. AFM is thus often employed to measure profiles of miscellaneous non-conductive samples, including two-dimensional materials ( $c f$. Fig. 2c,d). The resolving power and the stability of both STM and AFM setups are sufficient to measure material thicknesses below $1 \mathrm{~nm}$ even at ambient conditions. However, these measurements do not provide any information on the chemical nature of the analyte.

\subsection{Tip-enhanced Raman Spectroscopy}

While the more traditional far-field optical microscopy's spatial resolution is limited by the diameter of tightest focus (typically 200-300 nm), ${ }^{[28]}$ methods which circumvent this limitation have by now established themselves in the analytical sciences. These typically make use of the confinement of light in the optical near field of an emitter, the illuminated area being over two orders of magnitude smaller than what is achievable with confocal techniques. This emitter may either be a small aperture, but also antennas of opaque materials have proven viable for scanning near-field optical microscopy (SNOM). Since surface plasmon resonances on nanoparticles and nanometer-sized structures can enhance Raman scattering of molecules located within their near field - a phenomenon known from surface-enhanced Raman spectroscopy (SERS) - a useful technique has been developed about 20 years ago that promised to combine the high spatial resolution of SNOM with the chemical information available from SERS: tip-enhanced Raman spectroscopy (TERS). [29] TERS has been employed to optically visualize nanometer- and sub-nanometersized features, even as small as individual molecular orbitals based on their vibrational activity. This has so far only been reliably demonstrated in ultra-high vacuum (UHV) and at cryogenic temperatures. ${ }^{[30]}$

TERS measurements carried out in air and at room temperature have not yet reproducibly shown the spatial resolution necessary to chemically map sub-nanometer-sized structures. A rigorous proof of spatial resolution must always contain multiple coherent pixels showing similar spectral features independent of the probe scanning direction. Unfortunately, the spatial prolongation of signals stemming from contaminants on the tip, which are 'dragged' along the scanning coordinate, may inaccurately suggest oblong features on the sample, the locations of which are naturally irreproducible. Similar artefacts include rapidly and uncontrollably changing spectra, a common occurrence in ambient TERS with bare tips, where the enhancing metallic nanostructure is exposed to the environment. These unwanted signals may stem from changes in the tip geometry or from adsorption of organic volatiles to the probe and their degradation; both artefacts cause fluctuations and sudden changes in spectral intensity. ${ }^{[31,32]}$ This calls for great caution when interpreting such data. Last but not least, instrument drift may impede the recording of large highresolution TERS images at ambient conditions. Localizing and resolving nanodefects in two-dimensional polymers with ambient TERS therefore still remains challenging.

These problems could be addressed by performing the TERS experiment in an ultrapure aqueous solution of an electrolyte with external control of the electrical potential applied to the substrate and the tip, respectively. The relatively high thermal conductivity of water compared to air decelerates tip degradation processes originating from a heat-induced structural transformation of the enhancing site. ${ }^{[3]}$ Furthermore, coating this enhancing nanostructure on the SPM tip with a thin layer of a dielectric material (e.g. $\mathrm{SiO}_{2}$ ) exhibiting a high refractive index may help to protect the active nanoantenna from any exposure to ambient contaminants. [34]

After the first rational synthesis of a 2D-polymer at the air-water interface had succeeded in 2011, ${ }^{[35]}$ Schlüter and coworkers prepared a monolayer of a trianthracene monomer which they photopolymerized macroscopically in a Langmuir-Blodgett trough (see Fig. 1b,c). ${ }^{[13]}$ In this study, Opilik et al. demonstrated the viability of ambient TERS imaging as a tool to investigate molecularly thin 
covalent sheets by mapping an edge of the 2D-polymer with a pixel size of $125 \times 125 \mathrm{~nm}^{2}$ and explaining the preferential formation of dianthracene bridges via [4+4]-cycloadditions from the resulting spectra. ${ }^{[13]}$ Several subsequent reports from our laboratory have strengthened the view that ambient TERS is indeed suitable for the chemical identification and characterization of monolayers, including covalently bound sheets such as 2D-polymers, ${ }^{[12,22,36,37]}$ where the appearance of characteristic bands in the Raman spectrum hints at the formation or absence of covalent bonds between monomer molecules (see Fig. 3). Although the direct quantification of TERS spectra was deemed inapplicable due to the aforementioned fluctuations in intensity, peak ratios, averaged over large sample areas, can be employed to estimate the overall conversion within the polymer sheet (e.g. 90\%[37]). The architectures of accessible, large polymer sheets differ widely and range from imine linkages formed by condensation (loss of water, see Fig. 1a) ${ }^{[12,22]}$ and polycyclic moieties arising from electrocyclic reactions (see Fig. 1b,c) [13,36,37] to macromolecular planar coordination complexes (see Fig. 1d) ${ }^{[14,35]}$ although the latter have, to our knowledge, never been examined with TERS. Further notable observations from ambient TERS studies on these systems include the reproducible, spatially localized increase in signal intensity of a 2D-polymer sheet on an $\mathrm{Au}(111)$ step edge indicating a certain degree of pliability in that material; and a spatial variability in signal intensity reflecting the structural differences in a monomer layer on gold related to its method of preparation. ${ }^{[22]}$ There is significant experimental evidence, supported by theoretical considerations, that the orientation of the enhanced field along the tip axis relative to the polarizability of the analyte's normal modes of vibration plays an important role in determining which modes are subject to enhancement while others are suppressed. This argument is based on TERS 'surface selection rules' and helps to explain the above-mentioned observations (cf. Fig. 3). [22]

\section{Discussion}

As research on two-dimensional polymers constitutes a relatively new and promising field in synthetic and analytical chemistry, there is scientific interest in a vast range of properties and potential applications of these unique covalent sheets. The challenges that investigations on these intriguing materials bring with them are, however, often only met by the most sensitive, state-of-the-art analytical methods available. ${ }^{[38]}$ Here, the method of choice clearly depends on the question that needs to be addressed. While scanning probe microscopic methods such as ambient AFM and STM comfortably reveal the profile of such a sample with sub-nanometer vertical resolution and quite flexible lateral dimensions, typically from tens of micrometers down to a few nanometers, electron microscopy of a free-standing polymer sheet typically leads to rapid sample destruction and is much more challenging to perform. The presence of a mechanically stable
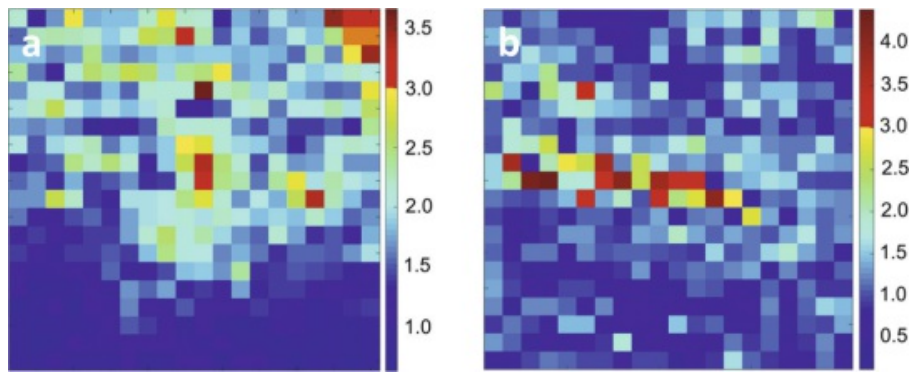

Fig. 3: Spatial distribution of the signal-to-noise ratio of the carboncarbon triple bond stretching mode in an STM-TERS measurements of a 2D-polymer sheet (cf. Figs. 1a and 2). The change in orientation of the triple bond affects the intensity of that band $\left(2220 \mathrm{~cm}^{-1}\right)$ according to the surface selection rule, and thus serves as a marker for defects. Pixel sizes: $5 \times 5 \mathrm{~nm}^{2}$ and $50 \times 50 \mathrm{~nm}^{2}$ for a) and b), respectively. ${ }^{[21,22]}$ thin film on a TEM grid after a suitable treatment of a monomer monolayer is nevertheless an established indication for the presence of a covalent sheet and the practice of showing a TEM image of the polymer is found throughout the literature, despite the difficulties associated with the measurement. After all, the diffraction pattern of the scattered electrons can hint at a structural regularity within the thin film, while AFM and STM are suitable to identify the presence of monolayers and to distinguish them from multilayered materials. Yet, neither electron microscopy nor scanning probe microscopy alone deliver enough structural information to characterize a thin film as a covalent sheet or even a crystalline two-dimensional polymer. Surely this structural information may be to some extent obtained for the bulk material by means of multidimensional nuclear magnetic resonance spectroscopy, infrared reflection absorption spectroscopy, high resolution electron energy loss spectroscopy, confocal Raman microscopy or X-ray methods, but these techniques are all lacking the spatial resolution to simultaneously locate and characterize the size and chemical nature of nanometer-size defect sites in the polymer monolayer. Tipenhanced Raman spectroscopy, on the contrary, has the potential to provide both structural and chemical information, meaning that it is conceptually suitable for insightful studies on 2D-polymers. A good understanding of the molecular structure of these materials has already been gained by employing this method (see section 2.4). Under ambient conditions, the analytical strength of TERS imaging is still limited by the lifetime of the tip and a trade-off between lateral resolution and spectral quality, which essentially stems from mechanical perturbations and drift. Based on its advantages, TERS is currently the method of choice for the chemical mapping of two-dimensional polymers at the nanoscale.

\section{Summary and Outlook}

The tremendous developments and improvements of analytical methods in the study of ultrathin materials of the past years have led to a significantly improved level of understanding regarding the chemistry and nanostructure of 2D-polymers. Crystallinity, a property any 2D-polymer must exhibit by definition, had initially been difficult to prove and has now been inferred from TERS images evidencing low-defect samples that are homogeneous over wide areas and show almost full conversion. Nanoscale defects in the polymer sheet can be localized with TERS imaging. If carried out in air, it currently offers a limit of detection for defects with sizes of less than $10 \mathrm{~nm}$. Today, TERS is establishing itself as an integral part of 2D-polymer analysis. For a swift initial control and an unequivocal characterization it should be supplemented by common methods such as microscopy and spectroscopy that deliver orthogonal information.

\section{Acknowledgements}

This project has received funding from the European Research Council (ERC) under the European Union's Horizon 2020 research and innovation programme under grant agreement No 741431 (2DNanoSpec). TN wishes to thank Prof. A. Dieter Schlüter for many helpful discussions and his invaluable support.

Received: May 23, 2019

[1] a) H. Staudinger, Ber. Dtsch. Chem. Ges. 1920, 53B, 1073, doi: 10.1002/ cber.19200530627; b) A. D. Jenkins, P. Kratochvíl, R. F. T. Stepto, U. W. Suter, Pure Appl. Chem. 1996, 68, 2287, doi: 10.1351/pac199668122287; c) L. G. Donaruma, B. P. Block, K. L. Loening, N. Platé, T. Tsuruta, K. C. Buschbeck, W. H. Powell, J. Reedijk, Pure Appl. Chem. 1985, 57, 149, doi: 10.1351/pac198557010149.

[2] a) A. Löfgren, A.-C. Albertsson, P. Dubois, R. Jérôme, J. Macromol. Sci., Rev Macromol. Chem. Phys. 1995, C35, 379, doi: 10.1080/15321799508014594; b) M. Karg, A. Pich, T. Hellweg, T. Hoare, L. A. Lyon, J. J. Crassous, D. Suzuki, R. A. Gumerov, S. Schneider, I. I. Potemkin, W. Richtering, Langmuir 2019, Just Accepted Manuscript, doi: 10.1021/acs.langmuir.8b04304. 
[3] M. Janata, B. Masař, L. Toman, P. Vlček, P. Látalová, J. Brus, P. Holler, React. Funct. Polym. 2003, 57, 137, doi: 10.1016/j.reactfunctpolym.2003.09.002.

[4] G. Mino, S. Kaizerman, J. Polym. Sci. 1958, 31, 242, doi: 10.1002/ pol.1958.1203112248.

[5] a) A. Bhattacharya, B. N. Misra, Prog. Polym. Sci. 2004, 29, 767, doi: 10.1016/j.progpolymsci.2004.05.002; b) K. L. Beers, S. C. Gaynor, K. Matyaszewski, S. S. Sheiko, M. Möller, Macromolecules (Washington, DC U.S.) 1998, 31, 9413, doi: 10.1021/ma981402i; c) D. A. Tomalia, A. M. Naylor, W. A. Goddard III, Angew. Chem., Int. Ed. Engl. 1990, 29, 138, doi: 10.1002/anie.199001381; d) A. D. Schlüter, J. P. Rabe in 'Encyclopedia of Polymer Science and Technology', 2001, p. 135, doi: 10.1002/0471440264. pst412; e) A. Zhang, A. D. Schlüter, Polym. Prepr. (Am. Chem. Soc., Div. Polym. Chem.) 2003, 44, 524.

[6] a) V. G. Taranenko, A. A. Trapeznikov, Kolloidn. Zh. 1979, 41, 380; b) X. Li, D. Zhang, K. Xiang, G. Huang, RSC Adv. 2014, 4, 32894, doi: 10.1039/ c4ra01877j; c) M. Akiba, A. S. Hashim, Prog. Polym. Sci. 1997, 22, 475, doi: 10.1016/S0079-6700(96)00015-9.

[7] a) A. Schumacher, L. Scandella, N. Kruse, R. Prins, Surf. Sci. 1993, 289 L595, doi: 10.1016/0167-2584(93)90727-Z; b) K. S. Novoselov, A. K. Geim, S. V. Morozov, D. Jiang, Y. Zhang, S. V. Dubonos, I. V. Grigorieva, A. A. Firsov, Science (Washington, DC) 2004, 306, 666, doi: 10.1126/science.1102896; c) J. R. Brent, N. Savjani, P. O’Brien, Prog. Mater. Sci. 2017, 89, 411, doi: 10.1016/j.pmatsci.2017.06.002; d) W. Choi, N. Choudhary, G. H. Han, J. Park, D. Akinwande, Y. H. Lee, Mater. Today (Oxford, U.K.) 2017, 20, 116, doi: 10.1016/j.mattod.2016.10.002; e) T. V. Khai, H. G. Na, D. S. Kwak, Y. J. Kwon, H. Ham, K. B. Shim, H. W. Kim, Nanotechnology 2013, 24, 145602, doi: 10.1088/0957-4484/24/14/145602.

[8] a) A. Pockels, J. W. S. B. Rayleigh, Nature 1891, 43, 437, doi: 10.1038/043437c0; b) I. Langmuir, K. B. Blodgett, Kolloid-Z. 1935, 73, 257, doi: 10.1007/BF01428777.

[9] V. Müller, A. Hinaut, M. Moradi, M. Baljozovic, T. A. Jung, P. Shahgaldian, H. Möhwald, G. Hofer, M. Kröger, B. T. King, E. Meyer, T. Glatzel, A. D. Schlüter, Angew. Chem., Int. Ed. 2018, 57, 10584, doi: 10.1002/ anie. 201804937

[10] G. Hofer, F. Grieder, M. Kröger, A. D. Schlüter, T. Weber, J. Appl. Cryst. 2018, 51, 481, doi: 10.1107/S1600576718002820.

[11] Figures reproduced with permission from Wiley-VCH Verlag GmbH \& Co. KGaA, refs [12,14] and the American Chemical Society, ref. [13].

[12] W. Dai, F. Shao, J. Szczerbiński, R. McCaffrey, R. Zenobi, Y. Jin, A. D. Schlüter, W. Zhang, Angew. Chem., Int. Ed. 2016, 55, 213, doi: 10.1002/ anie. 201508473 .

[13] L. Opilik, P. Payamyar, J. Szczerbiński, A. P. Schütz, M. Servalli, T. Hungerland, A. D. Schlüter, R. Zenobi, ACS Nano 2015, 9, 4252, doi: 10.1021/acsnano.5b00629.

[14] Z. Zheng, C. S. Ruiz-Vargas, T. Bauer, A. Rossi, P. Payamyar, A. Schütz, A Stemmer, J. Sakamoto, A. D. Schlüter, Macromol. Rapid Commun. 2013, 34, 1670, doi: 10.1002/marc.201300624

[15] a) H. Sahabudeen, H. Qi, B. A. Glatz, D. Tranca, R. Dong, Y. Hou, T. Zhang, C. Kuttner, T. Lehnert, G. Seifert, U. Kaiser, A. Fery, Z. Zheng, X. Feng, Nat. Commun. 2016, 7, 13461, doi: 10.1038/ncomms13461; b) I. Jung, M. Pelton, R. Piner, D. A. Dikin, S. Stankovich, S. Watcharotone, M. Hausner, R. S. Ruoff, Nano Lett. 2007, 7, 3569, doi: 10.1021/n10714177; c) S. Roddaro, P. Pingue, V. Piazza, V. Pellegrini, F. Beltram, Nano Lett. 2007, 7 , 2707, doi: 10.1021/nl0711581; d) P. Blake, E. W. Hill, A. H. Castro Neto, K. S. Novoselov, D. Jiang, R. Yang, T. J. Booth, A. K. Geim, Appl. Phys. Lett. 2007, 91, 063124, doi: 10.1063/1.2768624.

[16] a) M. Bruna, S. Borini, Appl. Phys. Lett. 2009, 94, 031901, doi: 10.1063/1.3073717; b) B. Mukherjee, F. Tseng, D. Gunlycke, K. K. Amara, G. Eda, E. Simsek, Opt. Mater. Express 2015, 5, 447, doi: 10.1364/ OME.5.000447.

[17] X. Wang, Y. P. Chen, D. D. Nolte, Opt. Express 2008, 16, 22105, doi: 10.1364/OE.16.022105.
[18] a) E. Ochoa-Martínez, M. Gabás, L. Barrutia, A. Pesquera, A. Centeno, S. Palanco, A. Zurutusa, C. Algora, Nanoscale 2015, 7, 1491, doi: 10.1039/ C4NR06119E; b) U. Wurstbauer, C. Röling, U. Wurstbauer, W. Wegscheider, M. Vaupel, P. H. Thiesen, D. Weiss, Appl. Phys. Lett. 2010, 97, 231901, doi 10.1063/1.3524226; c) S. Funke, B. Miller, E. Parzinger, P. Thiesen, A. W. Holleitner, U. Wurstbauer, J. Phys.: Condens. Matter 2016, 28, 385301, doi: 10.1088/0953-8984/28/38/385301.

[19] Z. Wang, I. S. Chun, X. Li, Z.-Y. Ong, E. Pop, L. Millet, M. Gillette, G. Popescu, Opt. Lett. 2010, 35, 208, doi: 10.1364/OL.35.000208.

[20] S. Khadir, P. Bon, D. Vignaud, E. Galopin, N. McEvoy, D. McCloskey, S. Monneret, G. Baffou, ACS Photonics 2017, 4, 3130, doi: 10.1021/ acsphotonics.7b00845.

[21] Figure reproduced with permission from the American Chemical Society, ref. [22].

[22] F. Shao, W. Dai, Y. Zhang, W. Zhang, A. D. Schlüter, R. Zenobi, ACS Nano 2018, 12, 5021, doi: 10.1021/acsnano.8b02513.

[23] E. Ruska, Z. Phys. 1934, 89, 90, doi: 10.1007/BF01333236.

[24] R. Erni, M. D. Rossell, C. Kisielowski, U. Dahmen, Phys. Rev. Lett. 2009 102, 096101, doi: 10.1103/PhysRevLett.102.096101.

[25] G. Algara-Siller, S. Kurasch, M. Sedighi, O. Lehtinen, U. Kaiser, Appl. Phys. Lett. 2013, 103, 203107, doi: 10.1063/1.4830036.

[26] a) G. Binnig, H. Rohrer, Surf. Sci. 1983, 126, 236, doi: 10.1016/00396028(83)90716-1; b) G. Binnig, H. Rohrer, Sci. Am. 1985, 253, 50, doi: 10.1038/scientificamerican0885-50.

[27] G. Binnig, C. F. Quate, C. Gerber, Phys. Rev. Lett. 1986, 56, 930, doi: 10.1103/PhysRevLett.56.930.

[28] J. Toporski, T. Dieing, O. Hollricher, 'Confocal Raman Microscopy', 2nd Edn., Springer International Publishing, 2018.

[29] a) R. M. Stöckle, Y. D. Suh, V. Deckert, R. Zenobi, Chem. Phys. Lett. 2000, 318, 131, doi: 10.1016/S0009-2614(99)01451-7; b) M. S. Anderson, Appl. Phys. Lett. 2000, 76, 3130, doi: 10.1063/1.126546; c) N. Hayazawa, Y. Inouye, Z. Sekkat, S. Kawata, Opt. Commun. 2000, 183, 333, doi: 10.1016/ S0030-4018(00)00894-4; d) B. Pettinger, G. Picardi, R. Schuster, G. Ertl, Electrochemistry (Tokyo, Jpn.) 2000, 68, 942.

[30] a) M. Liao, S. Jiang, C. Hu, R. Zhang, Y. Kuang, J. Zhu, Y. Zhang, Z. Dong, Nano Lett. 2016, 16, 4040, doi: 10.1021/acs.nanolett.6b00533; b) J. Lee, K. T. Crampton, N. Tallarida, V. A. Apkarian, Nature 2019, 568, 78, doi: 10.1038/s41586-019-1059-9.

[31] F. Shao, R. Zenobi, Anal. Bioanal. Chem. 2019, 411, 37, doi: 10.1007/ s00216-018-1392-0.

[32] J. Szczerbiński, L. Gyr, J. Kaeslin, R. Zenobi, Nano Lett. 2018, 18, 6740, doi: 10.1021/acs.nanolett.8b02426

[33] Z.-C. Zheng, S.-C. Huang, D.-Y. Wu, L.-Y. Meng, M.-H. Li, T.-X. Huang, J.-H. Zhong, X. Wang, Z.-L. Yang, B. Ren, J. Am. Chem. Soc. 2015, 137, 11928, doi: 10.1021/jacs.5b08143.

[34] a) J. F. Li, Y. F. Huang, Y. Ding, Z. L. Yang, S. B. Li, X. S. Zhou, F. R. Fan, W. Zhang, Z. Y. Zhou, D. Y. Wu, B. Ren, Z. L. Wang, Z. Q. Tian, Nature 2010, 464, 392; b) Y. P. Huang, S. C. Huang, X. J. Wang, N. Bodappa, C. Y. Li, H. Yin, H. S. Su, M. Meng, H. Zhang, B. Ren, Z. L. Yang, R. Zenobi, Z. Q. Tian, J. F. Li, Angew. Chem., Int. Ed. 2018, 57, 7523, doi: 10.1002/ anie. 201802892

[35] T. Bauer, Z. Zheng, A. Renn, R. Enning, A. Stemmer, J. Sakamoto, A. D. Schlüter, Angew. Chem., Int. Ed. 2011, 50, 7879, doi: 10.1002/ anie. 201100669

[36] F. Shao, V. Müller, Y. Zhang, A. D. Schlüter, R. Zenobi, Angew. Chem., Int. Ed. 2017, 56, 9361, doi: 10.1002/anie.201703800.

[37] V. Müller, F. Shao, M. Baljozovic, M. Moradi, Y. Zhang, T. Jung, W. B. Thompson, B. T. King, R. Zenobi, A. D. Schlüter, Angew. Chem., Int. Ed. 2017, 56, 15262, doi: 10.1002/anie.201707140.

[38] W. Wang, A. D. Schlüter, Macromol. Rapid Commun. 2019, 40, 1800719, doi: $10.1002 / \operatorname{marc} .201800719$ 